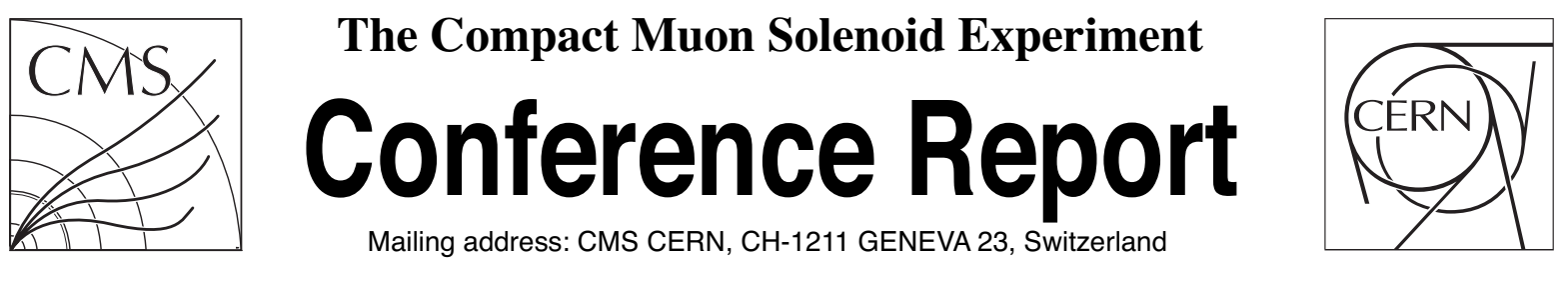

06 November 2009 (v2, 12 November 2009)

\title{
First Alignment of the Complete CMS Silicon Tracker
}

Kolja Kaschube for the CMS Collaboration

\begin{abstract}
We present the first results of the full CMS Silicon Tracker alignment based on several million reconstructed tracks from the cosmic data taken during commisioning runs with the detector in its final position. The all-silicon design of the CMS Tracker poses new challenges in aligning a complex system with 15148 silicon strip and 1440 silicon pixel modules. For optimal track-parameter resolution, the position and orientation of its modules need to be determined with a precision of several micrometers. For the modules well illuminated by cosmic ray particles, the ultimate precision has been achieved with data from the silicon modules traversed in-situ by charged muons used in combination with survey measurements. The achieved resolution in all five track parameters is controlled with data-driven validation of the track parameter measurements near the interaction region, and tested against prediction with detailed detector simulation.
\end{abstract}




\title{
First Alignment of the Complete CMS Silicon Tracker
}

\author{
Kolja Kaschube
}

\begin{abstract}
We present the first results of the full CMS Silicon Tracker alignment based on several million reconstructed tracks from the cosmic data taken during commisioning runs with the detector in its final position. The all-silicon design of the CMS Tracker poses new challenges in aligning a complex system with 15148 silicon strip and 1440 silicon pixel modules. For optimal track-parameter resolution, the position and orientation of its modules need to be determined with a precision of several micrometers. For the modules well illuminated by cosmic ray particles, the ultimate precision has been achieved with data from the silicon modules traversed in-situ by charged muons used in combination with survey measurements. The achieved resolution in all five track parameters is controlled with datadriven validation of the track parameter measurements near the interaction region, and tested against prediction with a detailed detector simulation.
\end{abstract}

Index Terms-Alignment, CMS, track detector, silicon strips, silicon pixels, cosmic muons.

\section{INTRODUCTION}

$\mathbf{T}$ HE Tracker [1] of the Compact Muon Solenoid (CMS) experiment at the Large Hadron Collider (LHC) is the largest silicon detector ever built. In order to fully exploit its capabilities, the position of all 16588 silicon modules must be known to a precision that is comparable to their intrinsic measurement resolution, i.e. less than ten micrometers. The determination of the positions and orientations of the modules and larger substructures of the Tracker is referred to as alignment.

In autumn of 2008, the CMS experiment recorded 270 million cosmic muons [2], 3.2 million of which were used to align the Tracker. The nominal CMS magnetic field of $3.8 \mathrm{~T}$ was in place during this data-taking period. Excellent performance was observed for all parts of the Tracker [3], [4].

In the following, the CMS Tracker is briefly introduced and the strategy that was used to achieve the alignment of the whole Tracker is presented. Finally, the alignment results are shown and discussed.

\section{THE CMS TRACKER}

The CMS Tracker consists of 15148 silicon strip modules and 1440 silicon pixel modules. From the nominal interaction point at the center of CMS, the Tracker extends $1.1 \mathrm{~m}$ in radial direction $(r)$ and $\pm 2.8 \mathrm{~m}$ along the beam pipe $(z)$. The azimuthal angle, $\phi$, is fully covered in the barrel region of the Tracker. The pixel modules are arranged in three barrel

K. Kaschube is with the Institute for Experimental Physics, University of Hamburg, Hamburg, Germany, e-mail: kolja.kaschube@ cern.ch

Manuscript received (ENTER DATE OF SUBMISSION). layers (BPIX) around the LHC beam pipe, as well as two disks on each side of the barrel (FPIX). The pixel modules have a size of $100 \times 150 \mu \mathrm{m}^{2}$ and measure both directions in the plane of the module, effectively producing a threedimensional hit measurement. Surrounding the pixels are four layers of silicon strip modules, called Tracker Inner Barrel (TIB), followed by another six layers, the Tracker Outer Barrel (TOB). There are three disks on either side of the TIB, referred to as Tracker Inner Disks (TIDs), followed by the endcaps (TECs) with nine disks each. Barrel layers and endcap disks are arranged concentrically around the beam axis such that the most sensitive coordinate is always in global $\phi$-direction. The strip modules consist of 512 or 768 strips with a pitch between 80 and $183 \mu \mathrm{m}$. They measure the coordinate perpendicular to the strips in the plane of the module. However, in the first two layers of both TIB and TOB as well as in several rings in the endcaps, two strip modules are glued together with a stereo angle of $100 \mathrm{mrad}$ (so-called "double-sided" modules), enabling a measurement in the direction of the strips. The intrinsic spatial resolution of the silicon modules is $10-30 \mu \mathrm{m}$.

In addition, a fixed Laser Alignment System [5] is installed in the strip detector. It comprises 40 infrared laser beams $(\lambda=$ $1075 \mathrm{~nm}$ ) which are directed onto 434 modules in TIB, TOB, and both TECs. The position of the beam spots on the modules can be determined with a precision of about $30 \mu \mathrm{m}$. Therefore, it is possible to monitor movements of the barrel parts and endcaps with respect to each other. The disks of each endcap can also be internally aligned.

\section{Alignment Strategy}

The knowledge of the alignment of a large silicon detector like the CMS Tracker is of utmost importance to the reconstruction performance of charged particle tracks. In reconstructing a trajectory of a particle traversing the detector, the distance of a measured hit on a module to the impact point of the predicted track (called "residual") is minimized. The sum of residuals of a track is minimal if the module positions are known with perfect precision. However, this is not the case in reality, of course. Therefore, the alignment of the modules is determined by minimizing the track residuals while keeping the module positions (henceforth called "alignment parameters") and track parameters free. The alignment parameters $\mathbf{p}$ are obtained after minimizing the expression

$$
\chi^{2}(\mathbf{p}, \mathbf{q})=\sum_{j}^{\text {tracks }} \sum_{i}^{\text {hits }} \mathbf{r}_{i j}^{T}\left(\mathbf{p}, \mathbf{q}_{j}\right) \mathbf{V}_{i j}^{-1} \mathbf{r}_{i j}\left(\mathbf{p}, \mathbf{q}_{j}\right),
$$

where $\mathbf{q}$ are the track parameters, $\mathbf{r}_{i j}$ are the residuals, and $\mathbf{V}_{i j}$ is the covariance matrix containing the hit errors. 
Two distinct algorithms were utilized to calculate the alignment parameters: a local iterative method called "Hits and Impact Points (HIP)" [6] and a global method called "Millepede II" [7]. The local method updates the alignment parameters after each iteration of Eq. (1) which makes it quite CPU timeconsuming as several tens of iterations are usually needed to converge to the best result. Also, correlations between Tracker modules are not taken into account within one iteration. On the other hand, this method uses the full CMS track model and includes survey measurements of the Tracker which were taken previously. The global method minimizes Eq. (1) in one step by estimating the alignment parameters and track quantities simultaneously. Thus, it needs less computing time than the local method. Correlations between modules are included, but a simplified track model, which did not include the scattering of particles within the Tracker material, was used as input.

The alignment methods were run several times in sequence, starting by aligning larger structures of the Tracker such as half-barrels and endcaps, then disks and layers, and ultimately calculating alignment parameters for all modules. While the results obtained with each method differed slightly, they were also consistent such that it was possible to run both methods in sequence to gain the best alignment.

Using the cosmic muon tracks recorded in 2008, the Tracker was aligned by initially running the global method a few times to solve global correlations between the modules and then applying the local method (again, in multiple steps) on the geometry obtained by the global method. The cosmic muons were required to have a momentum of at least $4 \mathrm{GeV} / \mathrm{c}$ and their reconstructed tracks had to have at least eight hits in the Tracker with a $\chi^{2} / n d f$, the $\chi^{2}$ per degree of freedom of the track fit, of less than six. Hits with large normalized residuals were removed from the fit. Out of the 3.2 million selected tracks, about 110000 traversed the pixel detector.

Optical survey measurements performed during the construction of the modules and installation of the Tracker provide initial alignment parameters for several parts of the detector. Module positions on their support structures, as well as the larger structures' relative positions, were measured in the inner strip detectors (TIB and TIDs), while only larger structures were studied in the TOB and TECs. The pixel detector was mapped in detail for modules and support structures.

\section{Alignment Results}

The alignment of the Tracker was carried out after ensuring that the calibration of the strip and pixel detectors was sufficient [3], [4]. This included the determination of the Lorentz angle and the alignment position errors.

The goodness of the track fit before and after aligning the Tracker with the cosmic muon tracks is shown in Fig. 1. The stand-alone results from the local and global methods are also included. The track prediction for each module was calculated by excluding the hit measurement from that module in order to avoid introducing a bias. A great improvement from the non-aligned geometry to the one obtained with the combined method can be seen.

Track residuals for all geometries are displayed in Fig. 2 for the sensitive coordinates in the Pixel Barrel and the Tracker

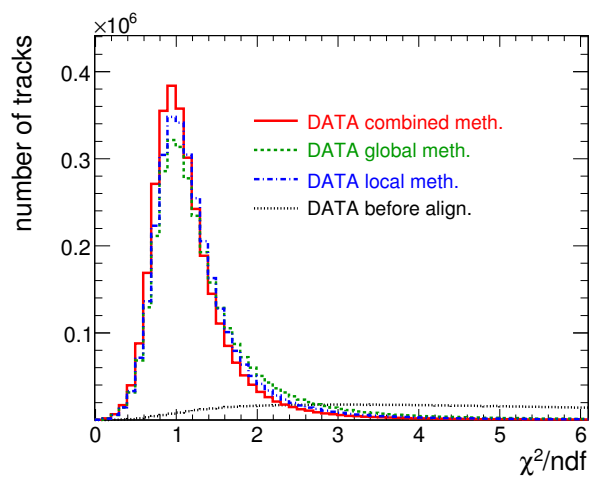

Fig. 1. Distribution of $\chi^{2}$ per degree of freedom for cosmic muon tracks before alignment (black dotted line), after alignment with the combined (red solid), global (green dashed), and local (blue dashed-dotted) methods.
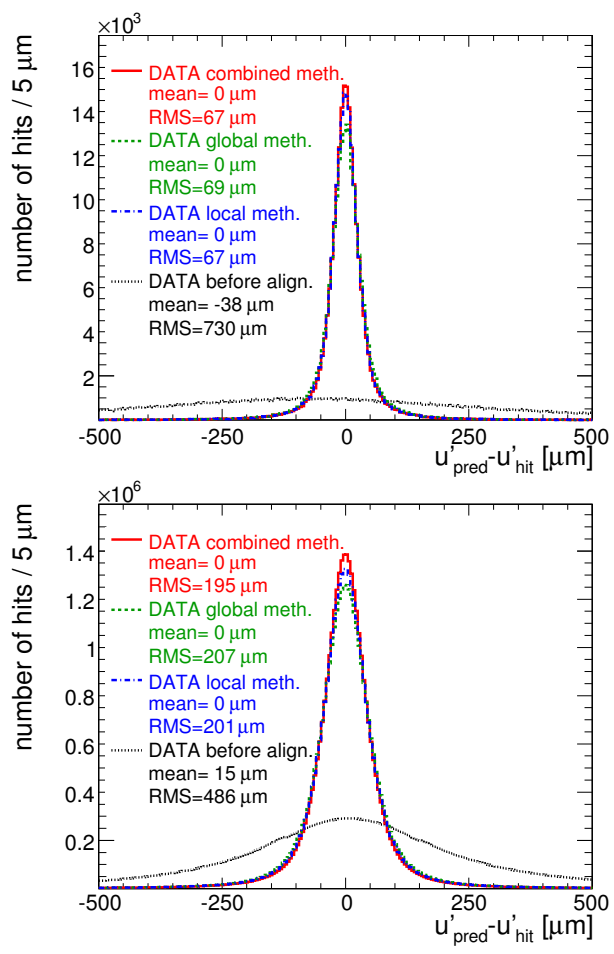

Fig. 2. Track residuals in the most sensitive coordinate for BPIX (top) and TOB (bottom) hits. Module positions are as known before alignment (black dotted line) and after alignment with the combined (red solid), global (green dashed), and local (blue dashed-dotted) methods.

Outer Barrel. These distributions are dominated by random effects such as multiple scattering and hit resolution, as well as systematic alignment errors. Therefore, the distribution of the median of the residuals (DMR) for each module were calculated and the corresponding root-mean-squared (RMS) values are quoted to determine the alignment precision. These are compared with Monte Carlo simulations using an ideal Tracker geometry and the geometry from the combined method. The DMR distributions for the Pixel Barrel and the Tracker Outer Barrel are shown in Fig. 3.

With the combined method, the RMS values of the most sensitive coordinates are about $3-4 \mu \mathrm{m}$ in the barrel parts and $3-14 \mu \mathrm{m}$ in the endcaps of the Tracker. Moreover, they are comparable to the results obtained with the ideal MC 

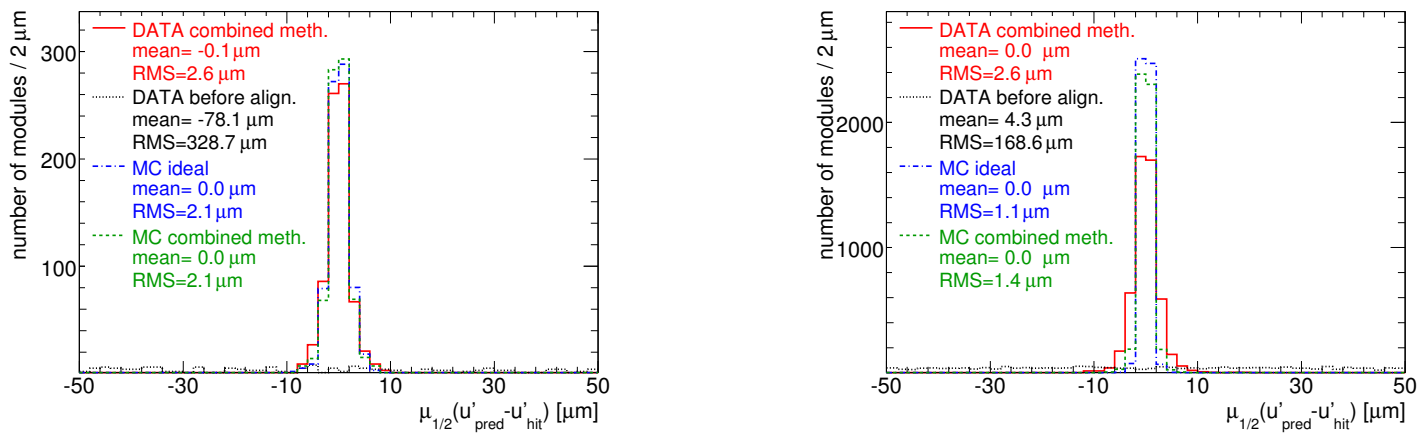

Fig. 3. Distribution of medians of residuals for modules with at least 30 hits, shown for BPIX (left) and TOB (right) before alignment (black dotted line), with the combined method and data (red solid), combined method and MC (green dashed), and ideal MC (blue dashed-dotted).

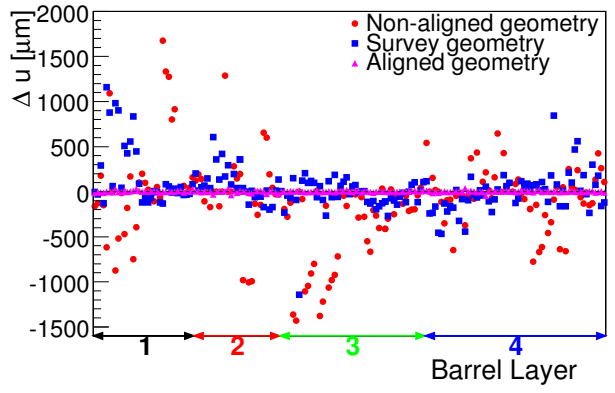

Fig. 4. Relative shift in the most sensitive coordinate of overlapping modules in the Tracker Inner Barrel; before alignment (red squares), from survey measurements (blue dots), with the combined method alignment (magenta triangles). Only modules with at least 100 hits located in the slice $80^{\circ}<\phi<100^{\circ}$ are shown.

simulation. The endcaps (FPIXs and TECs) were not aligned as precisely as the barrel due to the fact that cosmic muons penetrate the detector mostly vertically, which is parallel to the plane of the endcap modules. Since the hit reconstruction efficiency is very low for shallow incident angles, the amount of hits per module is considerably lower in the endcaps than in the barrel.

Another important method to validate alignment results is to use tracks crossing two modules within the same layer. All modules in the barrel overlap slightly with their neighbors so as to provide complete hermeticity in the azimuthal angle $(\phi)$. The difference between residuals of two overlapping hits gives a handle on the relative misalignment of the two modules in the sensitive coordinate. This is shown in Fig. 4 for modules in the Tracker Inner Barrel that have at least 100 hits in the overlap region. After aligning the Tracker with the combined method, the relative shift of overlapping modules with respect to this geometry was visibly reduced compared to non-aligned and survey geometries. This study was also performed with TOB modules and barrel pixels, yielding similar results, albeit only 35 hits were required in the pixel modules to account for their smaller size. The overlap method is assumed to be very precise since the hits are very close to each other, leading to little multiple scattering.

The track reconstruction performance on collision data can be estimated by splitting cosmic muon tracks at the point closest to the nominal interaction point and then refitting both legs as though they were collision tracks. Only tracks crossing the pixel detector were used and each leg was required to have at least three pixel hits. Track parameters such as transverse and longitudinal impact parameters, transverse momentum, and track angles were then compared for the two legs belonging to a cosmic track. Fig. 5 shows the transverse impact parameter and momentum differences, indicating that the aligned geometry allows for as precise a track reconstruction as is expected from MC simulations.

There are global deformations of the Tracker which may leave the $\chi^{2}$ of Eq. (1) unchanged after the alignment procedure. This means that alignment algorithms can rarely rectify these systematic misalignments which are accordingly called "weak modes." Global translations of the whole Tracker, for instance, can be resolved simply by defining (and fixing) the global coordinate system. Other distortions may not be discovered so easily, however. Therefore, nine systematic misalignments were examined: shifts in global $r, \phi$, and $z$ were applied to the geometry from the combined method, each as functions of the same three coordinates. Both alignment methods were used to re-align the systematically misaligned geometries. The outcome was then compared to the aligned geometry to gauge whether it was possible to recover from the systematic deformations. In Fig. 6, results from the global method for two weak modes in the Inner and Outer Barrel are shown. While the layer rotation $(r \Delta \phi$ vs. $r)$ can be almost completely recovered with cosmic muon tracks, a twist ( $r \Delta \phi$ vs. $z$ ) of the barrel is only partially recovered. In order to discover most of the misalignments introduced here, additional complementary data like collision tracks, beam halo muons, or information from the Laser Alignment System need to be combined in track-based alignment.

Finally, the Laser Alignment System was used to get an independent measure of the positions of TEC disks that was compared to the positions obtained with cosmic muon tracks and the combined method alignment. The disks were aligned in one rotational $(\phi)$ and two translational $(x$ and $y$ ) degrees of freedom within the disk plane as the LAS is insensitive to displacements orthogonal to the disk plane. 31 of 40 beams 

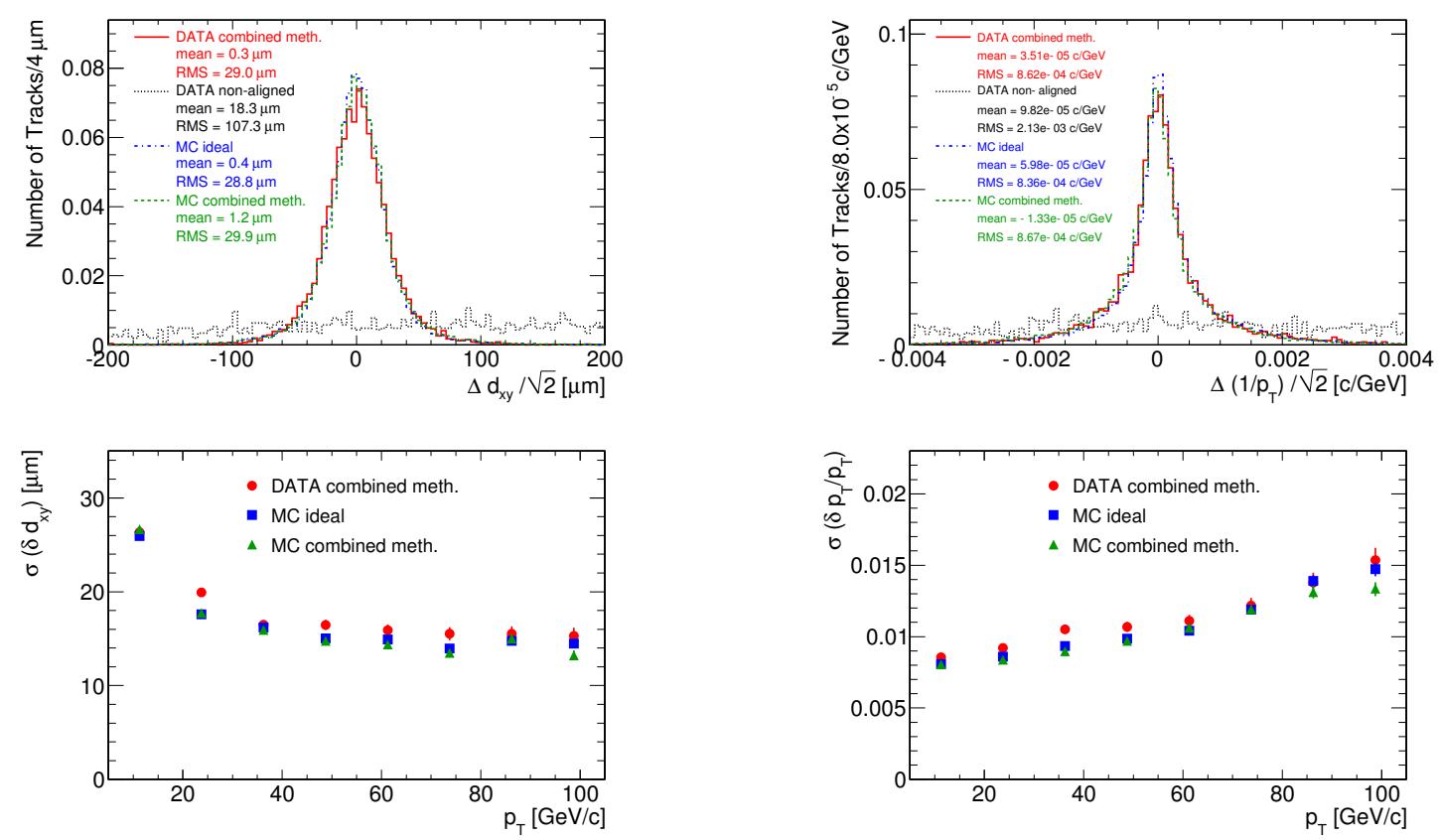

Fig. 5. Difference in track parameters of split cosmic track upper and lower legs. Top row: Transverse impact parameter difference $\Delta d_{x y}$ (left) and inverse transverse momentum difference (right) before alignment (black dotted line), with the combined method and data (red solid), combined method and MC (green dashed), and ideal MC (blue dashed-dotted). Bottom row: RMS of transverse impact parameter $d_{x y}$ (left) and RMS of transverse momentum resolution (right) vs. transverse momentum for data with the combined method (red dots), MC with combined method (green triangles), and ideal MC (blue squares).
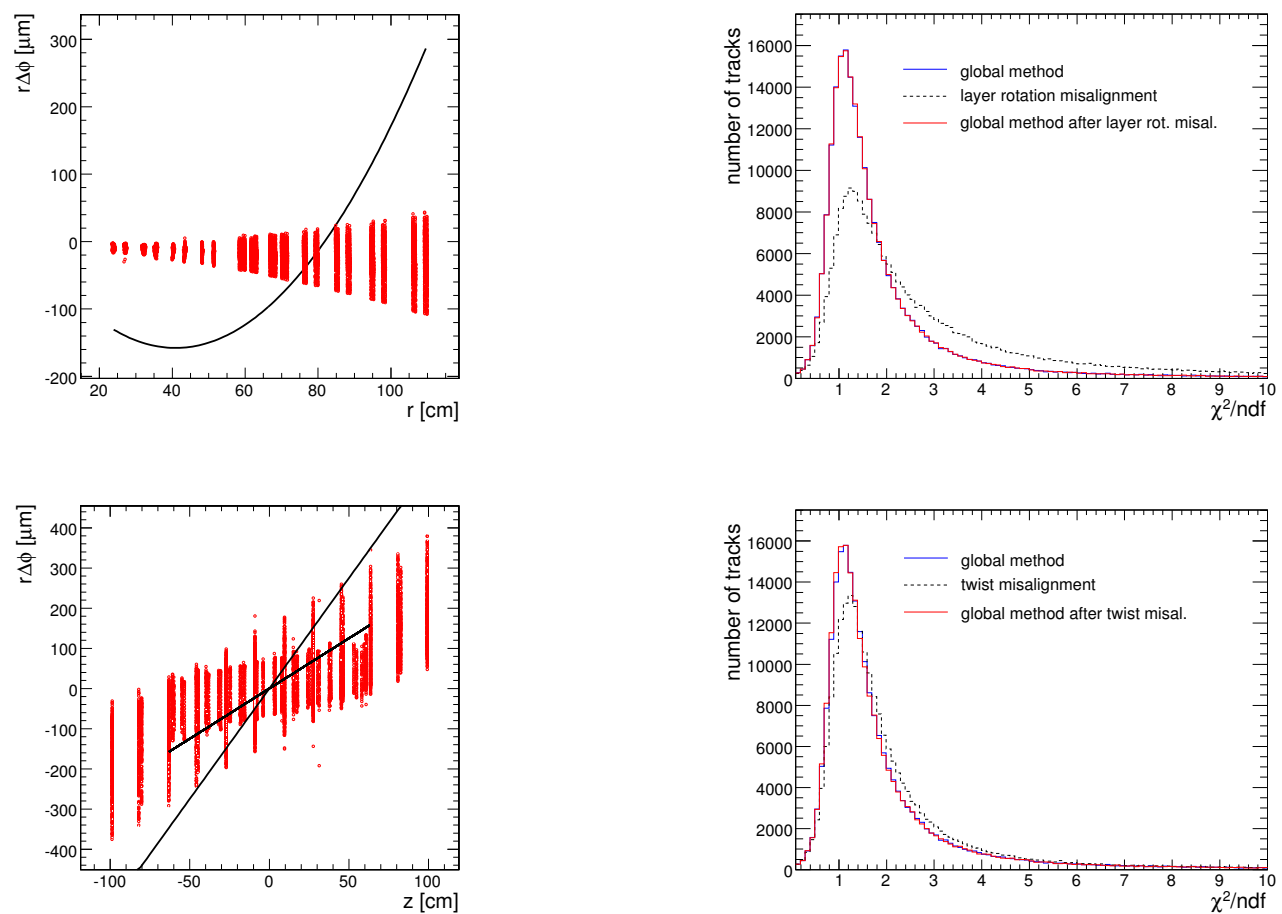

Fig. 6. Comparison of the position of TIB and TOB modules with respect to the geometry after alignment with the global method after applying systematic deformations (black solid lines) and after alignment (red dots), shown for layer rotation (top left) and twist (bottom left) misalignments. The corresponding $\chi 2 / n d f$ distributions of the tracks are displayed on the right after alignment with the global method (blue solid lines), after applying the misalignments (black dashed), and after re-aligning (red solid lines, close to blue solid lines). 
of the LAS were fully usable during the cosmic ray datataking in 2008. A good agreement was observed between LAS, combined method, and survey data.

\section{Conclusion}

The alignment of the complete CMS Tracker was performed with cosmic muon tracks recorded in 2008. The analysis was carried out with two alignment methods which were run in sequence to gain the best alignment precision. In the barrel parts of the Tracker, the precision was $3-4 \mu \mathrm{m}$ in the most sensitive coordinate, whereas the endcaps were aligned with a precision of $3-14 \mu \mathrm{m}$, as quoted from the RMS of the medians of residuals per module.

The track reconstruction performance was vastly improved with respect to the non-aligned geometry, and results are in agreement with detailed Monte Carlo simulations using a perfectly aligned Tracker. Some systematic distortions may still be present in the aligned geometry and it will take tracks from proton beam data to discover them. The Laser Alignment System was used to provide an independent cross-check of the positions of larger structures of the Silicon Strip Tracker.

The alignment gained from this exercise is being updated with cosmic ray data taken in 2009 and will be used in studies of first LHC collision data.

\section{ACKNOWLEDGMENT}

The author would like to thank his colleagues from the CMS Collaboration for producing the shown results and University of Hamburg for giving the opportunity to attend this conference. Additional thanks go out to BMBF and the Terascale Alliance for support and the organizers of the conference for their hospitality.

\section{REFERENCES}

[1] CMS Collaboration, "The CMS Experiment at the CERN LHC," JINST 0803 (2008) S08004.

[2] CMS Collaboration, "Commissioning of the CMS Experiment and the Cosmic Run at Four Tesla" CMS-CFT-09-008, to be submitted to JINST, 2009.

[3] CMS Collaboration, "The CMS Silicon Strip Tracker Operation and Performance with Cosmic Rays in 3.8 T Magnetic Field"CMS-CFT-09002, to be submitted to JINST, 2009.

[4] CMS Collaboration, "Performance of the CMS Pixel detector with cosmic ray data ," CMS-CFT-09-001, to be submitted to JINST, 2009.

[5] A. Ostapchouk, S. Schael, R. Siedling, B. Wittmer, "The Alignment System of the CMS Tracker," CMS NOTE-2001/053, 2001.

[6] V. Karimäki, T. Lampén, and F.-P. Schilling, "The HIP Algorithm for Track Based Alignment and its Application to the CMS Pixel Detector," CMS NOTE-2006/018, 2006.

[7] V. Blobel, "Software Alignment for Tracking Detectors," Nucl. Instr. Methods Phys. Res. A 566, 5, 2006.

Kolja Kaschube received his diploma in physics from the University of Hamburg, Hamburg, Germany. He is currently a graduate student at University of Hamburg working on alignment of the CMS Tracker and searches for new physics at the Large Hadron Collider. 